\title{
Saleh's Model of AM/AM and AM/PM Conversions is not a Model without Memory - Another Proof
}

\author{
Andrzej Borys
}

\begin{abstract}
The so-called Saleh's representation for description of the AM/AM and AM/PM conversions, occurring in communication power amplifiers, consists of two expressions that describe them as functions of a real-valued baseband signal modulating the carrier amplitude. It is a common view that this description forms a model without memory. We show here that the above belief is not correct; just the opposite is true. To prove this, we take into account an equivalent description of the Saleh's model called the quadrature model of bandpass nonlinearities and express it in a form of a nonlinear operator. Afterwards, we check whether this operator possesses a zero memory. To this end, we use an appropriate theorem of the nonlinear systems theory. Finally, as a result of this investigation, we observe that the memory of the above operator is nonzero.
\end{abstract}

Kyeywords-Saleh's model of AM/AM and AM/PM conversions, power amplifiers, memory effects, in-phase and quadrature representation of nonlinear devices

\section{INTRODUCTION}

$\mathrm{P}$ OWER amplifiers (PAs) used in wireless communications, as for example in satellite ones [1], exhibit nonlinear distortions in the operating regimes in which they work. These kinds of distortions manifest by occurrence of the so-called $\mathrm{AM} / \mathrm{AM}$ and $\mathrm{AM} / \mathrm{PM}$ conversions. More precisely, they are caused by the nonlinearities of amplifiers working in the transition area between their linear and nonlinear regions of operation, and also, slightly, in the nonlinear region.

There were presented in the literature many ways of modeling the AM/AM and AM/PM conversions occurring in the PAs. Here, we mention only a few of them [1-3], maybe most representative ones. An up-to-date survey of them was presented in a tutorial [4].

Saleh developed in [3] a simple but accurate model for description of the AM/AM and AM/PM conversions. It is one of the most frequently used and cited models. Note also that this model is assumed in the literature to belong to the group of memoryless ones. In other words, it does not incorporate memory effects.

Let us now describe briefly the Saleh's model in what follows, similarly as in [5]. We will do this after [3]. So, to this end, assume that the following bandpass input signal

The author is with the Department of Marine Telecommunications, Faculty of Electrical Engineering, Gdynia Maritime University, Gdynia, Poland (email: a.borys@we.am.gdynia.pl).

$$
x(t)=r(t) \cos \left(\omega_{c} t+\psi(t)\right)
$$

is applied to a PA. In (1), $\omega_{c}=2 \pi f_{c}$ with $f_{c}$ meaning the carrier frequency, $t$ denotes a time variable, and $j=\sqrt{-1}$. Moreover, the bandpass input signal $x(t)$ contains a slowly varying real-valued baseband signal $r(t)$ that modulates the carrier amplitude. Furthermore, the carrier phase changes with time according to a function $\psi(t)$. This function, similarly as $r(t)$, represents also a slowly varying baseband signal.

Then, after [3] (for more details see also, for example, [6]), the PA output signal can be expressed as

$$
y(t)=A(r(t)) \cos \left(\omega_{c} t+\psi(t)+\Phi(r(t))\right) .
$$

In (2), $A(r(t))$ and $\psi(t)+\Phi(r(t))$ denote the carrier amplitude and its phase, respectively, at the amplifier output. The function $A(r(t))$ is assumed to be a nonlinear function of $r(t)$. That is $A(r(t)) \neq a \cdot r(t)$ holds here, where $a$ stands for a real-valued constant. Moreover, it is assumed that an additional phase component $\Phi(r(t))$ in (2) does not equal zero and depends upon the slowly varying baseband signal $r(t)$. This means that a kind of the amplitude modulation expressed by the nonlinear characteristic $A(r(t))$ as well as the phase modulation expressed by another function $\Phi(r(t))$ occur here, and both are caused by the signal $r(t)$. Therefore, we refer to $A(r(t))$ as the AM/AM characteristic and to $\Phi(r(t))$ as the AM/PM characteristic.

In [3], Saleh developed a model, which approximates the above characteristics by the following functions

$$
A(r(t))=\frac{a_{1} r(t)}{1+a_{2}(r(t))^{2}}, \quad \Phi(r(t))=\frac{b_{1}(r(t))^{2}}{1+b_{2}(r(t))^{2}},
$$

where the coefficients $a_{1}$ and $a_{2}$ as well as $b_{1}$ and $b_{2}$ assume real values and need adjustment to the measured data for a given amplifier. 
Note now that (2) can be rewritten in an equivalent form as

$$
\begin{gathered}
y(t)=A(r(t)) \cos (\Phi(r(t))) \cos \left(\omega_{c} t+\psi(t)\right)- \\
-A(r(t)) \sin (\Phi(r(t))) \sin \left(\omega_{c} t+\psi(t)\right) .
\end{gathered}
$$

Next, it follows from (4) that the in-phase $p(t)$ and quadrature $q(t)$ components of the amplifier output signal $y(t)$ are given by

and

$$
p(t)=P(r(t)) \cos \left(\omega_{c} t+\psi(t)\right)
$$

$$
q(t)=-Q(r(t)) \sin \left(\omega_{c} t+\psi(t)\right)
$$

respectively. Further, the form of the functions $P(r(t))$ and $Q(r(t))$ in (5) and (6), accordingly, follows from (4) and (3). That is we have

$$
\begin{aligned}
& P(r(t))=A(r(t)) \cos (\Phi(r(t)))= \\
& =\frac{a_{1} r(t)}{1+a_{2}(r(t))^{2}} \cdot \cos \left(\frac{b_{1}(r(t))^{2}}{1+b_{2}(r(t))^{2}}\right)
\end{aligned}
$$

and

$$
\begin{aligned}
& Q(r(t))=A(r(t)) \sin (\Phi(r(t)))= \\
& =\frac{a_{1} r(t)}{1+a_{2}(r(t))^{2}} \cdot \sin \left(\frac{b_{1}(r(t))^{2}}{1+b_{2}(r(t))^{2}}\right) .
\end{aligned}
$$

The expressions (4), (5), and (6) were the basis of a model developed in [2] and in other publications in the 1970s, which preceded Saleh's article [3]. In these papers, the functions $P(r(t))$ and $Q(r(t))$ were approximated in a different way from that in [3], for example, by using polynomials or Bessel functions. The resulting model was called the quadrature model of bandpass nonlinearities [2] or simply the quadrature model.

One thing is worth noting in the above model, namely that both its components, the in-phase and quadrature one, depend upon the AM/AM and AM/PM conversions. This is immediately seen by looking at the relations (5-8). However, when the AM/PM conversion becomes zero, "the quadrature path" in the aforementioned model vanishes.

In [3], Saleh linked also his model with the quadrature one, exploiting the relations (7) and (8). In the rest of this paper, we will use a form of the above model that was used by Benedetto et al. in [1].

\section{QUADRATURE MODEL AND NONLINEAR OPERATOR DESCRIBING IT}

In [1], Benedetto et al. used the quadrature model to describe behaviour of a nonlinear device (as, for example, a power amplifier working in its nonlinear region of operation) exhibiting the AM/AM and AM/PM conversions. This model is visualized here in Fig. 1; it is also used by the author of this paper in a complementing work [5].

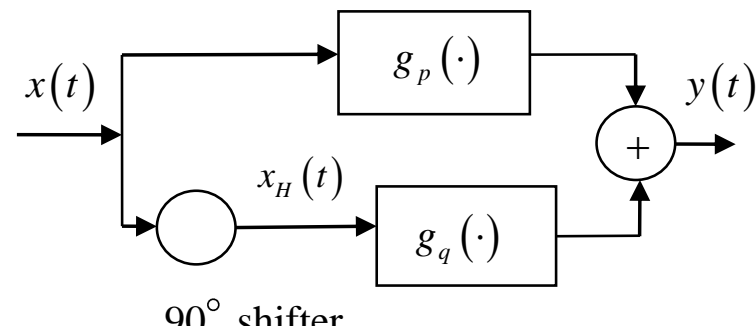

Fig. 1. The quadrature model of a nonlinear device exhibiting the AM/AM and AM/PM conversions.

The quadrature model of Fig. 1 consists of two parallel paths (branches). The upper one represents the model in-phase component, but the lower path its quadrature component, respectively. The first is described by a memoryless nonlinearity $g_{p}(\cdot)$, which can represented, for example, by a power series with real-valued coefficients. Similarly, the nonlinearity $g_{q}(\cdot)$ in the lower branch is assumed to be memoryless, too. So, it can be also modelled by a power series with real-valued coefficients, another one. However, the latter nonlinearity in Fig. 1 is preceded by a 90 degree shifter. And this makes the difference between the branches in Fig. 1.

Consider now the operation of shifting the signal $x(t)$ in Fig. 1 by 90 degrees to get another signal denoted as $x_{H}(t)$. This operation can be described in the time domain by the following convolution operation

$$
x_{H}(t)=\frac{1}{\pi} \int_{-\infty}^{\infty} \frac{x(\tau)}{t-\tau} d \tau
$$

The relation (9) describes the operation performed by the socalled Hilbert transformer, in the time domain. For more details regarding this topic, see, for example, [7].

Taking into account (9) and looking at Fig. 1, we see that the output signal $y(t)$ at the nonlinear device scheme of Fig. 1 is related with the input signal $x(t)$ by the relation

$$
\begin{gathered}
y(t)=g_{p}(x(t))+g_{q}\left(x_{H}(t)\right)= \\
=g_{p}(x(t))+g_{q}\left(\frac{1}{\pi} \int_{-\infty}^{\infty} \frac{x(\tau)}{t-\tau} d \tau\right) .
\end{gathered}
$$


Further, (10) can be expressed in the operator form as

$$
y(t)=N(x(t))=N_{1}(x(t))+N_{2}(x(t))
$$

with

$$
\begin{aligned}
& N_{1}(x(t))=g_{p}(x(t))= \\
& \quad=c_{1} x(t)+c_{2}(x(t))^{2}+c_{3}(x(t))^{3}+\ldots
\end{aligned}
$$

and

$$
\begin{aligned}
& N_{2}(x(t))=g_{q}\left(x_{H}(t)\right)= \\
& =d_{1} x_{H}(t)+d_{2}\left(x_{H}(t)\right)^{2}+d_{3}\left(x_{H}(t)\right)^{3}+\ldots= \\
& =\frac{d_{1}}{\pi} \int_{-\infty}^{\infty} \frac{x(\tau)}{t-\tau} d \tau+\frac{d_{2}}{\pi^{2}}\left(\int_{-\infty}^{\infty} \frac{x(\tau)}{t-\tau} d \tau\right)^{2}+ \\
& +\frac{d_{3}}{\pi^{3}}\left(\int_{-\infty}^{\infty} \frac{x(\tau)}{t-\tau} d \tau\right)^{3}+\ldots .
\end{aligned}
$$

By the way, note that (13) can be rewritten in the following form

$$
\begin{aligned}
& N_{2}(x(t))= \\
& =\frac{d_{1}}{\pi} \int_{-\infty}^{\infty} \frac{x(\tau)}{t-\tau} d \tau+\frac{d_{2}}{\pi^{2}}\left(\int_{-\infty}^{\infty} \frac{x(\tau)}{t-\tau} d \tau\right)^{2}+ \\
& +\frac{d_{3}}{\pi^{3}}\left(\int_{-\infty}^{\infty} \frac{x(\tau)}{t-\tau} d \tau\right)^{3}+\ldots=\frac{d_{1}}{\pi} \int_{-\infty}^{\infty} \frac{x(\tau)}{t-\tau} d \tau+ \\
& +\frac{d_{2}}{\pi^{2}} \int_{-\infty}^{\infty} \int_{-\infty}^{\infty} \frac{x\left(\tau_{1}\right)}{t-\tau_{1}} \frac{x\left(\tau_{2}\right)}{t-\tau_{2}} d \tau_{1} d \tau_{2}+ \\
& +\frac{d_{3}}{\pi^{3}} \int_{-\infty}^{\infty} \int_{-\infty}^{\infty} \int_{-\infty}^{\infty} \frac{x\left(\tau_{1}\right)}{t-\tau_{1}} \frac{x\left(\tau_{2}\right)}{t-\tau_{2}} \frac{x\left(\tau_{3}\right)}{t-\tau_{3}} d \tau_{1} d \tau_{2} d \tau_{3}+\ldots .
\end{aligned}
$$

And observe that the second series, equivalent to the first one in (14), is nothing else than a Volterra series [8]. Therefore, the operator $N_{2}(x(t))$ can be also called the Volterra operator [9] and, as such, it possesses memory [9]. The usage of the Volterra series to prove that the Saleh's model is not a model without memory is dealt with in [5], [10], and [11].

The operator $N(x(t))=(N x)(t)$ introduced above is defined by relations (11-13). (Note that $(N x)(t)$ stands for another useful form of notation used for operators.) This operator is a sum of two other operators $N_{1}(x(t))$ and $N_{2}(x(t))$. The former is, according to (12), expanded in a power series with real-valued coefficients $c_{1}, c_{2}, c_{3}$, and so on; we will see that it is memoryless. But the latter is a little bit more complicated; it is a composite operator. Its inner part means performing in the time domain the operation defined by the Hilbert transformer [7], but the outer one follows from the memoryless nonlinearity $g_{q}(\cdot)$, which is here (see (13)) expanded in a power series with real-valued coefficients $d_{1}$, $d_{2}, d_{3}$, and so on. Further, as we will see later, the operation performed by the Hilbert transformer is an operation with memory. So, altogether, in connection with the memoryless operation indicated by $g_{q}(\cdot)$, it makes the operator $N_{2}(x(t))$ to be an operator having memory. At this point, we remark that it is important to see that the operator $N_{2}(x(t))$, as indicated, works on the signal $x(t)$. In other words, it is an operator associated with the device input signal $x(t)$.

Remember now that the Saleh's model [3] and related ones like the quadrature model [3] or the quadrature model of bandpass nonlinearities [2] are assumed in the literature [4] to belong to the group of memoryless ones. That is they do not incorporate memory effects. In what follows, we will correct this common belief using the generic description for all the aforementioned models in form of the operator (or sum of operators), which was just developed above. We will do this by showing that this operator possesses nonzero memory. However, here, we will use another approach - different from that utilized in [5] - to achieve the aforementioned goal. In [5], a mathematical tool called the Volterra series [1] was used. But in this paper, we exploit some general results (theorems and lemmas) proved in the theory of operators. And with their use, we obtain the same result as the one got in [5]. Therefore, we can treat the derivations presented here as complementing the corresponding ones in [5] as well as strengthening them at the same time.

\section{CONSIDERATION OF LINEAR PART OF OPERATOR $N$}

To understand better the verification problem of whether a nonlinear operator possesses memory or does not have it, let us start with checking this on the linear part of the operator $N$ defined in the previous section. So, consider now the linear part of (11), which will be given by

$$
y_{l}(t)=N_{l}(x(t))=N_{1 l}(x(t))+N_{2 l}(x(t))
$$

with

$$
N_{1 l}(x(t))=g_{p l}(x(t))=c_{1} x(t)
$$

and

$$
\begin{gathered}
N_{2 l}(x(t))=g_{q l}\left(x_{H}(t)\right)=d_{1} x_{H}(t)= \\
=\frac{d_{1}}{\pi} \int_{-\infty}^{\infty} \frac{x(\tau)}{t-\tau} d \tau
\end{gathered}
$$

where the added index " $l$ " means a linear part of. 
In the next step, we invoke a theorem regarding representation of linear systems published by Sandberg in [12]. It has been shown in [13] that this theorem can be used in classification of linear systems or linear operators with respect to the memory held. Without going into details, the aforementioned theorem [12] and the discussion in [13] referred to as say the following:

Let $N_{l}$ be a linear, causal, and time-invariant operator (representation, map). Further, take a "windowing" operator $W_{\sigma}$ given by

$$
\left(W_{\sigma} x\right)(t)=w_{\sigma}(t) x(t)
$$

with the function $w_{\sigma}(t)$ having the following form

$$
w_{\sigma}(t)=\left\{\begin{array}{l}
1 \text { for }|t| \leq \sigma \\
0 \text { for }|t|>\sigma
\end{array},\right.
$$

where $\sigma$ means any positive number. Now, under the above assumptions, if

$$
\left(N_{l} x\right)(t)=\left(N_{l} W_{\sigma} x\right)(t)
$$

holds for every positive number $\sigma$; then the operator $N_{l}$ possesses no memory. That is it is a memoryless one.

In what follows, we will use the above statement to verify whether the following operators $N_{l}, N_{1 l}$, and $N_{2 l}$ occurring in (15) have memory or are memoryless. And let us start with the operator $N_{1 l}$. Obviously, we take a time while of verification or observation, say $t_{v}$, lying in the range of activity of the windowing function $w_{\sigma}(t)$. That is we choose $t_{v} \leq \sigma$. Note that for this time while we obtain $\left(N_{1 l} x\right)\left(t_{v}\right)=a_{1} x\left(t_{v}\right)$ for every $\sigma>0$ and $t_{v} \leq \sigma$. In the next step, see that for the same values of $\sigma>0$ and $t_{v} \leq \sigma$, we get $\left(N_{1 l} W_{\sigma} x\right)\left(t_{v}\right)=a_{1} x\left(t_{v}\right)$ because then $\left(W_{\sigma} x\right)\left(t_{v}\right)=x\left(t_{v}\right)$ holds. So, the condition (20), i.e. $\left(N_{1 l} x\right)\left(t_{v}\right)=\left(N_{1 l} W_{\sigma} x\right)\left(t_{v}\right)$, is fulfilled in this case. Finally, this means that the operator $N_{1 l}$ is memoryless.

Now, consider the operator $N_{2 l}$. Obviously, as before, we choose a time while of verification $t_{v} \leq \sigma$. That is such time while which lies in the range of activity of the windowing function $w_{\sigma}(t)$. Observe now that we get

$$
\left(N_{2 l} x\right)\left(t_{v}\right)=\frac{d_{1}}{\pi} \int_{-\infty}^{\infty} \frac{x(\tau)}{t_{v}-\tau} d \tau
$$

from (17). However, we have

$$
\begin{aligned}
& \left(N_{2 l} W_{\sigma} x\right)\left(t_{v}\right)=\frac{d_{1}}{\pi} \int_{-\infty}^{\infty} \frac{w_{\sigma}(\tau) x(\tau)}{t_{v}-\tau} d \tau= \\
& =\frac{d_{1}}{\pi} \int_{-\sigma}^{\sigma} \frac{x(\tau)}{t_{v}-\tau} d \tau
\end{aligned}
$$

for the value of the composite operator $N_{2 l} W_{\sigma}$ at the time while $t_{v}$ by virtue of the function $w_{\sigma}(t)$ given by (19).

Comparison of the right-hand sides of (21) and (22) shows that they contain different expressions. Let us check whether their values differ for the time while $t_{v}$. To this end, let us evaluate their difference $\Delta_{2 l}$ by taking into account (21) and (22). That is we will consider

$$
\begin{aligned}
& \Delta_{2 l}=\left(N_{2 l} x\right)\left(t_{v}\right)-\left(N_{2 l} W_{\sigma} x\right)\left(t_{v}\right)= \\
& =\frac{d_{1}}{\pi} \int_{-\infty}^{\infty} \frac{x(\tau)}{t_{v}-\tau} d \tau-\frac{d_{1}}{\pi} \int_{-\sigma}^{\sigma} \frac{x(\tau)}{t_{v}-\tau} d \tau .
\end{aligned}
$$

Next, observe that (23) can be rewritten as

$$
\begin{aligned}
& \Delta_{2 l}=\left(N_{2 l} x\right)\left(t_{v}\right)-\left(N_{2 l} W_{\sigma} x\right)\left(t_{v}\right)= \\
& =\frac{d_{1}}{\pi} \int_{-\infty}^{-\sigma} \frac{x(\tau)}{t_{v}-\tau} d \tau+\frac{d_{1}}{\pi} \int_{\sigma}^{\infty} \frac{x(\tau)}{t_{v}-\tau} d \tau .
\end{aligned}
$$

Note that the function $x(t)$ occurring in (24) means any function. So, let us choose for our purpose of testing a function $x(t)$, which is defined by

$$
x_{\text {test }}(t)=\left\{\begin{array}{l}
1 \text { for }|t| \leq 3 \sigma \\
0 \text { for }|t|>3 \sigma
\end{array} .\right.
$$

Substituting the function given by (25) into (24) gives

$$
\begin{aligned}
& \Delta_{2 l}=\left(N_{2 l} x_{\text {test }}\right)\left(t_{v}\right)-\left(N_{2 l} W_{\sigma} x_{\text {test }}\right)\left(t_{v}\right)= \\
& =\frac{d_{1}}{\pi} \int_{-3 \sigma}^{-\sigma} \frac{1}{t_{v}-\tau} d \tau+\frac{d_{1}}{\pi} \int_{\sigma}^{3 \sigma} \frac{1}{t_{v}-\tau} d \tau .
\end{aligned}
$$

Using the following

$$
\int \frac{1}{t_{v}-\tau} d \tau=-\ln \left|\tau-t_{v}\right|
$$


in (26), we get from (26)

$$
\begin{aligned}
\Delta_{2 l} & =\frac{d_{1}}{\pi}\left[\ln \left|-3 \sigma-t_{v}\right|-\ln \left|-3 \sigma-t_{v}\right|+\right. \\
& \left.+\ln \left|\sigma-t_{v}\right|-\ln \left|3 \sigma-t_{v}\right|\right] .
\end{aligned}
$$

Obviously, there are such values of $\sigma$ and $t_{v}$ for which $\Delta_{2 l} \neq 0$. This fact is sufficient to conclude that the operator $N_{2 l}$ possesses memory.

Finally in this section, consider the operator $N_{l}$ that according to (11) is a sum of operators $N_{1 l}$, and $N_{2 l}$ analysed just before. So, by virtue of this, we have

$$
\begin{aligned}
& \Delta_{l}=\left(N_{l} x\right)\left(t_{v}\right)-\left(N_{l} W_{\sigma} x\right)\left(t_{v}\right)= \\
& =\left(N_{1 l} x\right)\left(t_{v}\right)-\left(N_{1 l} W_{\sigma} x\right)\left(t_{v}\right)+ \\
& +\left(N_{2 l} x\right)\left(t_{v}\right)-\left(N_{2 l} W_{\sigma} x\right)\left(t_{v}\right)
\end{aligned}
$$

or in a more detailed form

$$
\begin{aligned}
& \Delta_{l}=\left(N_{l} x\right)\left(t_{v}\right)-\left(N_{l} W_{\sigma} x\right)\left(t_{v}\right)= \\
& =c_{1} x\left(t_{v}\right)-c_{1} x\left(t_{v}\right)+ \\
& +\frac{d_{1}}{\pi} \int_{-\infty}^{\infty} \frac{x(\tau)}{t_{v}-\tau} d \tau-\frac{d_{1}}{\pi} \int_{-\sigma}^{\sigma} \frac{x(\tau)}{t_{v}-\tau} d \tau .
\end{aligned}
$$

From (30), we get

$$
\begin{aligned}
\Delta_{l} & =\left(N_{l} x\right)\left(t_{v}\right)-\left(N_{l} W_{\sigma} x\right)\left(t_{v}\right)= \\
& =\left(N_{2 l} x\right)\left(t_{v}\right)-\left(N_{2 l} W_{\sigma} x\right)\left(t_{v}\right)= \\
& =\frac{d_{1}}{\pi} \int_{-\infty}^{\infty} \frac{x(\tau)}{t_{v}-\tau} d \tau-\frac{d_{1}}{\pi} \int_{-\sigma}^{\sigma} \frac{x(\tau)}{t_{v}-\tau} d \tau= \\
= & \Delta_{2 l} .
\end{aligned}
$$

But, as shown above, $\Delta_{2 l} \neq 0$ holds for many combinations of values of $\sigma$ and $t_{v}$. Therefore, the operator $N_{l}$ possesses memory. In other words, this is not a memoryless operator.

\section{CONSIDERATION OF NONLINEAR OPERATOR $N$}

Using the results obtained in the previous section and applying the same methodology, it can be shown that the nonlinear operator $N$ possesses the memory like its linear part $N_{l}$. On the other hand, this seems to be obvious because the strictly nonlinear part of $N$ considered here does not have any property to remove the memory from the linear part of $N$.

\section{SUMMARY}

This is the third paper in a series of three, together with [5] and [11], in which the problem of possessing memory by the Saleh's model of AM/AM and AM/PM conversions is discussed. In all of these works, we have proved that this model is not a memoryless one. Further, note that to show the above fact we have used three different approaches and exploited two different mathematical tools: the Volterra series [8], [9] and Sandbergs's theorem [12], [13]. In all of the three cases, we have arrived at the same result.

Finally, we draw the reader's attention to the fact that above conclusions regard also the models related with the Saleh's one, like the quadrature model [3] and the quadrature model of bandpass nonlinearities [2]. These are also models possessing memory.

\section{REFERENCES}

[1] S. Benedetto, E. Biglieri, and R. Daffara, "Modeling and performance evaluation of nonlinear satellite links. - A Volterra series approach", IEEE Trans. Aerospace Electron. Syst., vol. 15, pp. 494-507, 1979.

[2] A.R. Kaye, D.A. George, and M.J. Eric, "Analysis and compensation of bandpass nonlinearities for communications", IEEE Trans. on Commun. Technol., vol. 20, pp. 365-372, Oct. 1972.

[3] A. A. M. Saleh, "Frequency-independent and frequency-dependent nonlinear models of TWT amplifiers", IEEE Trans. on Communications, vol. 29, pp. 1715-1720, 1981.

[4] J. Joung, C. K. Ho, K. Adachi, and S. A. Sun, "Survey on poweramplifier-centric techniques for spectrum- and energy-efficient wireless communications", IEEE Communications Surveys \& Tutorials, vol. 17 pp. 315-333, 2015.

[5] A. Borys, "Quadrature mapping, Saleh's representation, and memory models", to be published in Int. Journal of Telecommunications and Electronics (JET), vol. 62, no. 4, pp. xx-xx, 2016.

[6] M. Jeruchim, P. Balaban, and K. Sam Shanmugan, Simulation of Communication Systems: Modeling, Methodology, and Techniques. New York: Kluwer, 2002.

[7] A. Borys, Nonlinear Aspects of Telecommunications: Discrete Volterra Series and Nonlinear Echo Cancellation, Boca Raton, Florida, USA: CRC Press, 2000.

[8] J. J. Bussgang, L. Ehrman, and J. W. Graham, "Analysis of nonlinear systems with multiple inputs," Proceedings of the IEEE, vol. 62, pp. 1088-1119, 1974.

[9] S. Boyd and L. O Chua, "Fading memory and the problem of approximating nonlinear operators with Volterra series", IEEE Trans. on Circuits and Systems, vol. 32, pp. 1150-1161, 1985.

[10] A. Borys and W. Sieńko, "On nonlinear distortions in satellite communication links and their equalization", in Proceedings of the 1st International Conference on Innovative Research and Maritime Applications of Space Technology IRMAST, Gdańsk, Poland, April 23 24, 2015, pp. $161-166$.

[11] A. Borys and W. Sieńko, "On modelling AM/AM and AM/PM conversions via Volterra series," Int. Journal of Telecommunications and Electronics (JET), vol. 62, pp. 267-272, 2016.

[12] I. W. Sandberg, "Bounded inputs and the representation of linear system maps", Circuits, Systems, and Signal Processing, vol. 24, pp. 103-115, 2005.

[13] A. Borys, "Sandberg's representation theorem and classification of linear systems", IEEE Trans. on Circuits and Systems - II: Express Briefs, vol. 55, pp. 678- 679, 2008. 